\title{
Design on Students Score Management System based on Asp.net
}

\author{
Zhe $\mathrm{Li}^{1, \mathrm{a}}$, Jiahui Wang ${ }^{2, \mathrm{~b}}$, Shuang Wei, \\ ${ }^{1}$ College of International Exchange, Bohai University, Jinzhou, 121013, China \\ ${ }^{2}$ College of Information Science and Technology, Bohai University, Jinzhou, 121013, China \\ ${ }^{3}$ School of Education and Sports, Bohai University, Jinzhou, 121013, China \\ a503412508@qq.com, b24351108@qq.com, cwsbd2000@163.com
}

\begin{abstract}
Keywords: Asp.net; students score management; system design; MVC framework; database design; functional design
\end{abstract}

\begin{abstract}
Score management is an important work for educational administration, and the important content of education informatization construction. In order to improve the level of teaching management, and provide a full range of information services for teachers and students in colleges and universities, this article is based on Asp.net framework design student achievement management system. First, Research on the Asp.net MVC basic processing procedure and illustrate the application of the model, view and controller. Then, carries on the logic structure design, database design results by "Students information table", "Course information table" and "Score information table" and other three tables; Finally, a functional design, following the abstract and incremental refinement, the principle of modularity and information hiding design all the functions of the system. In the actual development process, it can be adjusted according to actual situation of local content appropriate to make the system more in line with the actual demand.
\end{abstract}

\section{Introduction}

Teaching informationization is the core content of education informationization. Teaching is the center of the field of education, teaching information including teaching science and technology, education management informatization, spread of education informationization and modernization of teaching methods. Education informatization require in the education process more comprehensive use of computer, multimedia and network communication based on modern information technology, promote the education reform, so as to adapt to the coming of information society puts forward new requirements, to deepen the education reform and the implementation of quality education is of great significance.

Educational administration management system management system is an important part of education informatization, and student achievement management system is one of the core functions. Achievement is the result of the learning level and inspection, linked to various awards and assessment mechanism, relationship to the immediate interests of the students, even change the student's life trajectory, is of great significance to strengthen the academic record management [1]. Today's performance management is not the traditional meaning of the test scores, but need according to different subjects and professional division, the reasonable use weighted algorithm, the comprehensive quality of students, based on the results of the learning process and learning, through rigorous method to calculate the reasonable results are obtained. In order to meet this demand, in this paper, the integrated use of computer technology, database technology and Internet technology design student achievement management system.

\section{Asp.net MVC Framework}

Framework technology offers a wide range of software reuse, for developers to provide a unified software development kit, frees from tedious codes, this paper studies the business logic of the application, and shielding the underlying code implementation details, so as to improve development efficiency. In order to meet the needs of different applications, can be on the basis of 
the application and analysis, to improve and expand the function of the framework. The MVC (Model - View - Controller), the Model, View and Controller, an application of the input, the business logic and output according to the Model, View, Controller, an application is divided into a Model layer, View layer, control layer [2, 3]

The basic process of Asp.net MVC is the URL request coming from the client, find the value of the Controller and the Action will be asked to transfer to the Controller. Controller gain data Model objects, and passing the Model to the View, the View is responsible for rendering the page. Intuitively understandable as follows: the Model is access to web pages needed data, the Controller is to get the data, then data binding to the page control business processes, the Controller is ASP.NET PageLoad method of logic, the View is the aspx page. Process is shown in Fig.1.

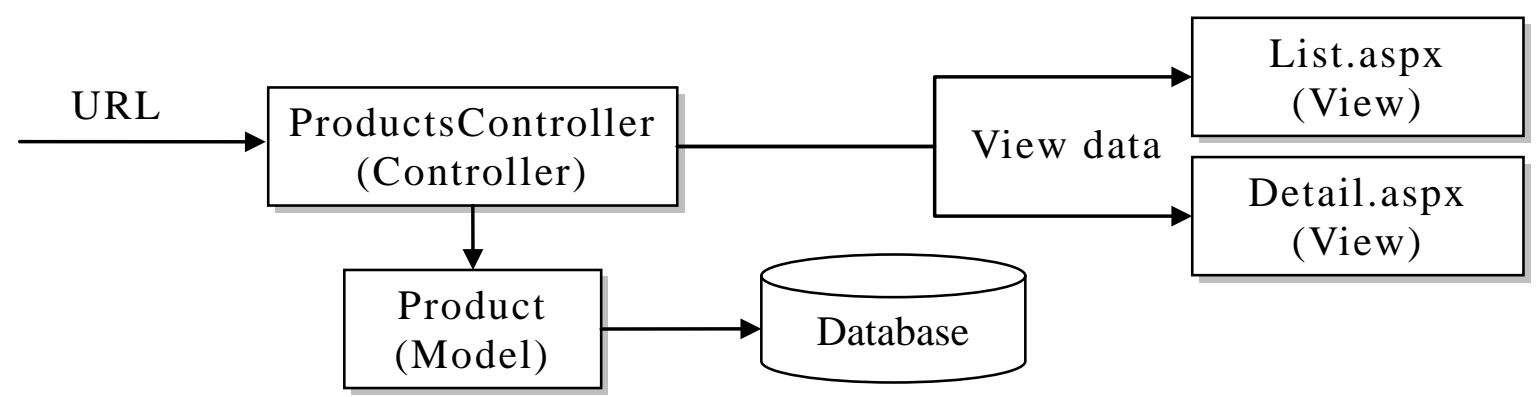

Fig. 1. Asp.net MVC process flow

Asp.net model [4]. It is implemented in the application data domain logic. The model object retrieval and storage condition in the database. Product object retrieves information from a database, for example, the operating data, and then write the updated information back to the database table.

Asp.net view [5]. It is to show the application user interface of the UI components. In general, to create the UI based on the model data. Based on the current state of the Product object, for example, edit the Product table view, display text boxes, drop-down list, and check boxes, and so on.

Asp.net controller is used to handle user interaction, work with the model, and select a view to render the user interface. In the MVC application, the view is used to display the information, the controller handles and responds to user input and interaction.

Many Asp.net MVC advantages, mainly displays in: by dividing the project into Model, View and Controller, complex projects easier maintenance, reduce the coupling between the project; Does not use view state and server form controls, can be more easily to control the behavior of the application; Application by the Controller to control the application request, and provide a native UrlRouting function to rewrite the Url; Make a Web application support for unit testing more wonderful; Superior performance in team development mode.

\section{Database Design}

Data in the database according to certain data model organize, description and storage, with smaller data redundancy, high independence and extensibility, and can provide all kinds of users to share. From small single transaction processing to the complex software system, mostly use advanced database technology to keep the system data integrity, integrity, and sharing. Database design is to create the database and application technology of the system. Database design is for a given application environment, constructing the optimal database model, establish a database and application systems, to store data effectively and meet the application requirements of various users.

Common database design includes three stages: first, the concept design phase, is the key to database design, through comprehensive, induction and abstraction of user requirements, to form an independent of the specific concept model of database management system; The second is, the logical design phase, the concept structure into a database management system supported by the data model, and carries on the optimization; Three is that the physical design phase, as the logical model to select a most suitable for the physical structure of the application environment, mainly the design of the storage structure and access method. Limited space, this article only carries on the 
logical structure design.

Logical structure design depends on the database management system. This article is based on Microsoft SQL Server to carry on the design of the company. Microsoft SQL Server is a comprehensive database platform, using the integrated business intelligence tools provides enterprise data management. Microsoft SQL Server database engine for relational data and structured data provides a more secure and reliable storage capabilities, to build and manage for the business of high availability and high performance data application. In SQL Server database engine is used to store, process, and protect the data of core services. Using the database engine can control access and quickly handle affairs, so as to satisfy the business enterprise inside very demanding and requires application need to process large amounts of data. Student achievement management system includes a "Students", "Course" and "Score" three entities, such as two entities exists between two contact "One - to - the things", that is, a student can be elective courses, a student has Many subjects, but a Course result corresponds to only One student [6, 7].Converts the three entities to table the logical structure of the name "Students information table", "Course information table" and "Score information table", as shown in Table 1.

Table 1. Database logical structure on students score management system

\begin{tabular}{|c|c|c|c|c|c|c|c|}
\hline \multicolumn{4}{|c|}{ Students information table } & \multicolumn{4}{|c|}{ Course information table } \\
\hline No & FieldName & DataType & Width & No & FieldName & DataType & Width \\
\hline 1 & StudentID & varchar & 10 & 21 & CourseID & varchar & 10 \\
\hline 2 & StudentName & varchar & 50 & 22 & CourseNumber & varchar & 10 \\
\hline 3 & CollegeNumber & varchar & 10 & 23 & CourseName & varchar & 50 \\
\hline 4 & CollegeName & varchar & 50 & 24 & OpenCollege & varchar & 50 \\
\hline 5 & ProfessionalNumber & varchar & 10 & 25 & CourseCredit & decimal & 6,2 \\
\hline 6 & ProfessionalName & varchar & 50 & 26 & ExaminingMode & varchar & 20 \\
\hline 7 & ClassName & varchar & 50 & 27 & CourseIntroduction & varchar & $\max$ \\
\hline 8 & EntryYear & smalling & 2 & \multicolumn{4}{|c|}{ Score information table } \\
\hline 9 & BirthDate & datetime & 4 & No & FieldName & DataType & Width \\
\hline 10 & MailingAddress & varchar & 100 & 31 & StudentID & varchar & 10 \\
\hline 11 & Postalcode & char & 6 & 32 & CourseNumber & varchar & 10 \\
\hline 12 & E-mail & varchar & 50 & 33 & CourseName & varchar & 50 \\
\hline 13 & ContactTelephone & varchar & 50 & 34 & ExaminationDate & datetime & 4 \\
\hline 14 & AverageScore & decimal & 6,2 & 35 & UsualScore & decimal & 6,2 \\
\hline 15 & Ranking & smalling & 2 & 36 & ExamScore & decimal & 6,2 \\
\hline 16 & Note & varchar & $\max$ & 37 & TotalScore & decimal & 6,2 \\
\hline
\end{tabular}

\section{Functional Design}

System function design is conducted after the feasibility study and demand analysis, is the core of the profile design work. Functional design should follow the profile design of abstract, incremental refinement, principles of modularity and information hiding, etc.

Abstraction is the concentration and sum up the similar aspects, ignored the differences between them. That is, the abstract is to take out the essential characteristics of things, and temporarily don't consider the details. Modular design of the software can have different level of abstraction. At the highest levels of abstraction, you can use the problems of environment language description method. While on the lower level of abstraction, it will take procedural methods.

Stepwise refinement is a kind of top-down design strategy, the software architecture, according 
to the top-down approach, details and data details the process of all levels of refinement gradually, until can statements in a programming language to realize, finally established the whole architecture of the software. Set a functional refinement or detailed information, gives the implementation details, divided into several elements, and then these ingredients are detailed. With the refinement of work gradually, designers can get more and more details.

Module is part of the program logic can be separated. Module has a certain function, can be individually named after the collection of data and program statements to be accessed. If the module is divided into independent of each other, module becomes smaller, the less the amount of workload each module. However, when the decomposition module number increases, the greater contact between modules, these modules link up to the amount of work also will increase.

Information hiding, is refers to the implementation details of each module is hidden to other modules. That is, included in a module of information (including process and data), do not allow the other don't need these information module USES. Adopts the principle of information hiding design software for testing and maintenance during the period of software modification work provides great convenience.

Using the above principle, with reference to relevant literature [8, 9], the design of system function is shown in Fig. 2.

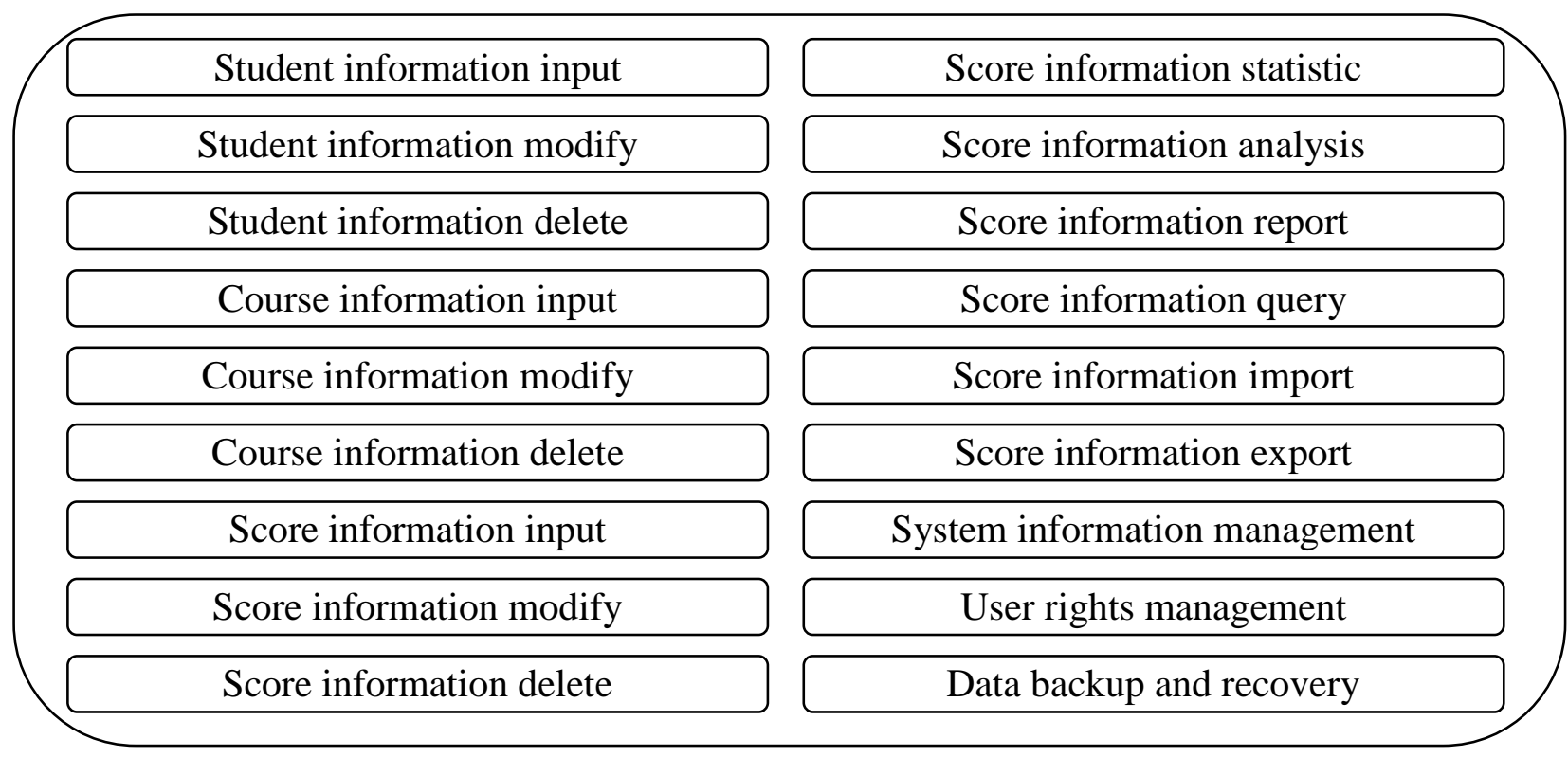

Fig. 2. Function on students score management system

\section{Conclusion}

Student achievement management as daily work of educational administration in colleges and universities, schools, colleges, teachers and students involved many factors, such as the reform of teaching management system, especially the universally implement the credit system in universities and elective system, maintain day-to-day management and student performance management work increasingly multifarious, urgently needs the operation is simple, powerful function, low cost and has the humanized student achievement management system [10]. This article is based on the target design, in the actual development process and adjusted according to actual situation of local content appropriate to make the system more in line with the actual demand.

\section{Acknowledgement}

This work is supported by social science fund project of Liaoning province (L14DGL044): Research on Effect evaluation and optimization mechanism for urban and rural compulsory education teacher balanced allocation policy; "Twelfth Five Year" planning project of Liaoning 
education and science (JG15ZXY15): Research on Effect evaluation and optimization mechanism for policy implementation of "special post teachers" in rural areas; 2016 annual economic and social development project in Liaoning province (2016lslktzixlx-01): Formation mechanism and countermeasure research the classroom' phubbing in colleges and universities in Liaoning.

\section{References}

[1] M. Y. Yin, "Design and Implementation of Student Achievement Management System Based on B/S Structure," Master's Degree of Jilin University, 2015.

[2] M. Xu, "The Triple Master Business Management System Design and Implement Based On the SSH2 Framework of MVC Mode," Master's degree of Ocean University of China, 2010.

[3] Dragos-Paul Pop, Adam Altar, "Designing an MVC Model for Rapid Web Application Development," Procedia Engineering, vol. 69, no. 1, pp. 1172-1179, 2014.

[4] Q. F. Wang, B. B. Hu, "Research on ASP.NET 4 MVC Framework Based on Multi-tiers Architecture," Computer \& Telecommunication, vol. 21, no. 10, pp. 64-66, 2015.

[5] D. L. Huang, "Research on Web Development Based on ASP.NET MVC Framework," Journal of Henan Science and Technology, vol. 40, no. 7, pp. 26-27, 2015.

[6] Z. W. Yang, "Designation and implementation of College Student Achievenent Management System," Master's Degree of University of Electronic Science and Technology of China, 2013.

[7] Z. Wang, "The Design and implementation of Student Achievenent Management System," Master's Degree of University of Electronic Science and Technology of China, 2013.

[8] Q. Y. Hu, "Design and Implementation of Student Score Managemente," Master's Degree of Jilin University, 2015.

[9] X. Z. Tang, H. P. Liu, X. L. Lan, "Thinking of the development and practice of performance inquiry system based on ASP.NET environment," China CIO News, vol. 18, no. 4, pp. 28, 2015.

[10] H. Lang, "Design and implementation of college student achievement management system based on.NET," Master's Degree of Xi'an Electronic and Science University, 2013. 